\title{
Searching for the best evidence. Part 2: searching CINAHL and Medline
}

The first part of this editorial (July issue) described the information resources that nurses need to support their practices and other activities. This editorial will focus on 1 type of information resource-large bibliographic databases-and will describe how to harness the full potential of 2 databases (CINAHL and Medline) to identify studies that report high quality research that is ready for clinical application.

Proverbially speaking, looking for studies that are ready for clinical application is like looking for a needle in a haystack. The healthcare literature contains an abundance of research reports and studies that are in the early stages of development but few studies that are appropriate for clinical application. One estimate is that for every 5000 ideas that are initially postulated as improvements in health care, only 1 is fully evaluated and proved to be effective for changing healthcare practice. ${ }^{1}$ This makes effective and efficient searching of the literature even more critical.

Fortunately, articles reporting findings that are ready for clinical application can be retrieved using search strategies based on the principle of searching for studies which use the right method to answer their research question. We will briefly describe the research methodologies used in high quality studies on treatment (interventions), diagnosis (screening and assessment), prognosis, causation, as well as systematic reviews and qualitative studies as described in the purpose and procedure section, and relate these to terms and phrases that can be applied for searching CINAHL and Medline. The methodologies described in articles that are ready for clinical application are unique and separate these articles from news reports, idea papers, laboratory and animal studies, early human research, and clinical research that does not use the most appropriate methodology for the question. The table summarises search terms that can be used to retrieve primary (ie, original) studies and systematic reviews. Many of the strategies for searching Medline have been validated by comparing the results with those from handsearches of journals-the gold standard. ${ }^{2}$ Searches using these tested strategies are likely to produce fewer irrelevant citations. Comparable methodological strategies for searching CINAHL have not been formally assessed, although this is planned. Consequently, the CINAHL search strategies will retrieve a broader range of articles than the Medline strategies and may be less likely to find only studies that used appropriate methodologies.

\section{Treatment (interventions)}

Treatment studies are the most common type of "ready for clinical application" studies. The "best" evidence of treatment effectiveness comes from randomised controlled and clinical trials and from systematic reviews that combine the results of these primary studies. In addition to treatment and intervention studies, prevention, control, and quality improvement issues are studied using the same methodology. High quality treatment trials are often blinded (ie, researchers, clinicians, and assessors do not know which intervention a participant has received) and should have a minimum of $80 \%$ follow up of participants at the end of the study. Indexers for CINAHL and Medline assign index terms (known as Medical Subject Headings or MeSH in Medline) for randomised controlled trials, clinical trials, blinding, and placebos but not for follow up. Authors of articles often refer to "randomisation," "blinding," "placebos," and "follow up" in their abstracts and titles and these terms can be used in textword searching. Neither the indexing done by the staff that produce CINAHL and Medline nor descriptions by authors are perfect or consistent, and therefore the most effective search strategy will involve a combination of assigned index terms and textwords. For example, to answer a question about the effectiveness of sucrose for reducing pain in newborn infants who are having a painful procedure, a Medline search using the content terms sucrose, pain, and newborn infant (sucrose [index term] AND pain [index term] AND infant, newborn [index term]) retrieves 14 articles. Adding in search terms related to treatment methodology (clinical trial [publication type] OR random: [textword] OR double-blind method [index term] OR placebo: [textword]) limits the retrieval to 11 high quality studies. All 11 of the articles (10 original studies and 1 meta-analysis) are relevant.

Whereas these citations mainly refer to original studies, systematic reviews collect original studies, synthesise the results across the studies, and derive an overall "answer" to the question posed. Using the systematic review search strategy in the table-it can be used for all categories of problems-Medline retrieves 1 systematic review on the topic. A systematic review is often a better place to start looking for answers to clinical questions than original studies.

The Cochrane Library, described in the previous editorial, is another rich source of citations to randomised controlled trials. It includes more than 100000 citations to clinical trials from Medline, CINAHL, and other bibliographic databases and handsearches of many healthcare journals. It also has a large collection of full systematic reviews done by members of the Cochrane Collaboration and a bibliographic database of other systematic reviews collected by the NHS Centre for Reviews and Dissemination at the University of York in the UK.

\section{Diagnosis (screening and assessment)}

Nurses spend considerable time assessing patients and applying the principles of screening and diagnosis, and increasingly they are responsible for describing the test and explaining results to patients. Most of the high quality studies in this category are indexed in Medline with terms related to the evaluation of diagnostic tests such as "sensitivity" and "specificity." CINAHL indexing terms and policies reflect a more general approach and the index term "diagnosis," when exploded (ie, when all subdivisions of the indexed term 
are retrieved), covers most aspects of nursing assessment, screening (people with no symptoms or indications of disease), and diagnosis (people with symptoms or conditions suggestive of disease or disorders). The methodological terms for "diagnosis" are embedded within this "explosion" (ie, "explode diagnosis" retrieves citations indexed under "sensitivity and specificity" and "predictive value of tests").

For example, a CINAHL search to identify studies that address the question how useful are screening and diagnostic tests for postpartum depression? using the terms "post partum depression" [index term] AND "explode diagnosis" [index term] retrieves 32 articles. Most deal with postpartum depression; some are only marginally related to our question, and some are not clinical studies, which probably reflects the lack of validated search strategies in CINAHL. A study by Stamp et al assessed the Modified Antenatal Screening Questionnaire that identified women in Australia who were vulnerable to becoming depressed after childbirth. ${ }^{3}$ It was a valuable start to developing a high quality screening instrument, but the questionnaire had relatively high rates of missing a diagnosis in women who were depressed and misdiagnosing women who were not. The authors concluded that more research is needed.

\section{Prognosis}

Prognosis research is concerned with the natural history and prognosis of a disease or condition. Natural history deals with the progression of a disease, beginning at its biological onset (when there may not be any signs or symptoms) whereas prognosis deals with the progression of disease after diagnosis. A nursing related prognosis question might be what information about prognosis might inform discussions with a woman who has an HIV positive baby? A natural history study (found in both CINAHL and Medline) done by Barnhart et al starts with infants born to mothers with HIV infection. ${ }^{4}$ Chil- dren were followed up until they developed the disease and died. On average, children lived to a mean age of 9.4 years but developed moderate symptoms in their second year of life. Prognosis studies are more common than natural history studies. High quality prognosis studies are usually prospective cohort studies. Search terms related to prognosis may include "incidence," "prevalence," "mortality," "morbidity," and "disease progression."

\section{Causation}

Causation questions such as does passive smoking cause respiratory problems in children? and does hormone replacement therapy protect against Alzheimer's disease? can be studied using several methodologies. Randomised controlled trials and cohort studies are strong methodologies, but sometimes cannot be used (eg, one cannot randomise children to experience passive smoking). Many more case control studies exist, but they are more susceptible to bias. Cross sectional studies with statistically adjusted group analysis are the most common type of causation studies, but are the weakest of the 4 study designs because data about exposure and outcome are collected simultaneously and therefore causal inferences cannot be made. Search terms in the table reflect these methodologies.

\section{Systematic reviews}

Individual primary studies from any of the categories above can be collected and analysed in a systematic review. The systematic reviews that are considered to be "evidence based" and ready for use in clinical situations must have a clearly stated purpose, a description of methods used to identify studies, and inclusion and exclusion criteria for selecting studies for analysis. Strategies for retrieval of systematic reviews must include terms that gather meta-analyses plus

Methodological search strategies for CINAHL and Medline

\begin{tabular}{|c|c|c|}
\hline Area of Research & Terms for searching CINAHL & Terms for searching Medline \\
\hline Treatment (interventions) & $\begin{array}{l}\text { Explode clinical trials (index term) OR random: } \\
\text { (textword) OR research (publication type) }\end{array}$ & $\begin{array}{l}\text { *Clinical trial (publication type) OR random: (textword) OR } \\
\text { double-blind method (index word) OR placebo: } \\
\text { (textword) }\end{array}$ \\
\hline Diagnosis (screening and assessment) & Explode diagnosis (index term) & $\begin{array}{l}\text { *Explode sensitivity and specificity (index term) OR } \\
\text { sensitivit: (textword) OR specificit: (textword) OR } \\
\text { diagnosis (subheading exploded) OR diagnostic use } \\
\text { (subheading exploded) }\end{array}$ \\
\hline Prognosis & $\begin{array}{l}\text { Disease progression (index term) OR explode } \\
\text { prospective studies (index term) OR explode } \\
\text { mortality (index term) OR explode morbidity (index } \\
\text { term) OR time factors (index term) OR explode } \\
\text { prognosis (index term) OR prognosis }(\text { textword) }\end{array}$ & $\begin{array}{l}\text { *Incidence (textword) OR explode mortality (index word) } \\
\text { OR follow-up studies (index word) OR prognosis: } \\
\text { (textword) OR predict: (textword) OR course: (textword) }\end{array}$ \\
\hline Causation & $\begin{array}{l}\text { Explode prospective studies (index term) OR explode } \\
\text { case-control studies (index term) OR explode risk } \\
\text { factors (index term) OR risk: (textword) }\end{array}$ & $\begin{array}{l}\text { *Explode cohort studies (index word) OR explode risk } \\
\text { (index word) OR odds ratio: (textword) OR relative risk: } \\
\text { (textword) OR case-control (textword) }\end{array}$ \\
\hline $\begin{array}{l}\text { Systematic reviews and meta-analyses }{ }^{5} \\
\text { Use these strategies in each of the categories } \\
\text { above for retrieval of systematic reviews }\end{array}$ & $\begin{array}{l}\text { Meta-analysis (index term) OR meta-analysis } \\
\text { (textword) OR metaanalysis (textword) OR } \\
\text { MEDLINE (textword) AND [review (publication type) } \\
\text { OR systematic review (textword)] }\end{array}$ & $\begin{array}{l}\text { Meta-analysis (publication type) OR meta-analysis (index } \\
\text { term) OR meta-analysis (textword) OR metaanalysis } \\
\text { (textword) OR systematic review: (textword) OR } \\
\text { [MEDLINE (textword) AND review (publication type)] }\end{array}$ \\
\hline Qualitative studies & $\begin{array}{l}\text { Qualitative studies (index word) OR ethnographic } \\
\text { research (index word) OR ethnonursing research } \\
\text { (index term) OR phenomenological research (index } \\
\text { term) OR focus groups (index term) OR grounded } \\
\text { theory (index term) }\end{array}$ & $\begin{array}{l}\text { Nursing methodology research (index term) OR } \\
\text { qualitative research (textword) OR grounded theory } \\
\text { (textword) OR ethnograph: (textword) }\end{array}$ \\
\hline
\end{tabular}

*These search strategies have been validated using handsearches of journals. See Haynes $e t a .^{2}$ Other search strategies have not been tested and will retrieve citations with a broad range of methodological quality.

Many searching platforms exist (please see part 1 of this editorial). You will need to check your searching system to "translate" designations such as publication type and index term into the proper searching form (eg, variations in punctuation).

“" or “*” is used to retrieve alternate endings in various systems (eg, "random:” will retrieve random, randomly, randomized, and randomised in the OVID system).

"Textword" refers to words that appear in the titles and abstracts of articles.

"Explode" retrieves all the subdivisions of an index term (eg, "explode sports" retrieves citations on bicycling, swimming, baseball, basketball, etc).

"Publication type" is a term assigned by database indexers to designate the form of the citation (eg, clinical trial, practice guideline). 
systematic reviews that are not meta-analyses-both index words and textwords are needed because indexing is not comprehensive enough to retrieve all categories of systematic reviews.

\section{Qualitative studies (understanding people and situations)}

Qualitative studies are important to clinicians and researchers alike. The editors of Evidence-Based Nursing and other collaborators are developing quality appraisal criteria for qualitative studies. Qualitative research often involves the collection of data through observation or indepth interviews with individuals or groups. These observations or interviews are carefully and comprehensively analysed and the results are presented in the form of understandings of the topic studied or the development of a theory. Qualitative study designs include, among others, ethnography, grounded theory, and phenomenology. The table includes suggestions for searching. Groups in the United States and Europe are working to develop retrieval techniques for these types of studies, and we will provide additional information as it becomes available.

\section{Conclusion}

Retrieving sound, high quality articles from CINAHL and Medline is necessary for keeping up to date. It can be a time consuming and frustrating process. Using methodology based search strategies, nurses can harness these databases to produce timely retrievals of high quality studies. The Cochrane Library is also a rich source to supplement searching in the large bibliographic databases described here.

Please give us your comments on searching. We will be happy to provide more help with information retrieval as it becomes available.

K ANN McKIBBON, MLS

Health Information Research Unit

SUSAN MARKS, BA, BEd

McMaster University

Hamilton, Ontario, Canada

1 Matson E. Speed kills (the competition). Fast Company 1996 Aug/Sep:84-91. 2 Haynes RB, Wilczynski N, McKibbon KA, et al. Developing optimal search strategies for detecting clinic
Inform Assoc 1994;1:447-58.

3 Stamp GE, Williams AS, Crowther CA. Predicting postnatal depression among pregnant women. Birth 1996;23:218-23.

4 Barnhart HX, Caldwell MB, Thomas P, et al, and the Pediatric Spectrum of Disease Clinical Consortium. Natural history of human immunodeficiency virus disease in perinatally infected children: an analysis from the pediatric spectrum of disease project. Pediatrics 1996;7:710-6.

5 Hunt DL, McKibbon KA. Locating and appraising systematic reviews. Ann Intern Med 1997;126:532-8.

\section{How to cite material from Evidence-Based Nursing}

The citation styles given below can be used when citing material that was published in Evidence-Based Nursing.

Citation FOr MATERIAL From AN EDitorial

- Mulhall A. Nursing, research, and the evidence [editorial]. Evidence-Based Nursing 1998 Jan;1:4-6.

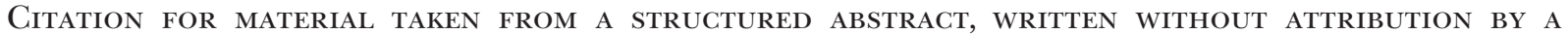
STAFF MEMBER

- Sucrose and commercially available milk reduced crying in newborns during a blood collection procedure [abstract]. Evidence-Based Nursing 1998 Jan;1:10. Abstract of: Blass EM. Milk-induced hypoalgesia in human newborns. Pediatrics 1997 Jan;99:825-9.

CitATION FOR MATERIAL TAKEN FROM A COMMENTARY TO AN ARTICLE.

- Carlisle C. Commentary on "A conceptual model described how adults responded to a simulated literacy assessment." Evidence-Based Nursing 1998 Jan;1:29. Comment on: Brez SM, Taylor M. Assessing literacy for patient teaching: perspectives of adults with low literacy skills. J Adv Nurs May;25:1040-7. 